\title{
Biometric Iris Recognition Based on Hybrid Technique
}

\author{
Khattab M. Ali Alheeti \\ Department of Information Systems, Anbar University College of Computer, Iraq \\ kattab1978@yahoo.com
}

\begin{abstract}
Iris Recognition is one of the important biometric recognition systems that identify people based on their eyes and iris. In this paper the iris recognition algorithm is implemented via histogram equalization and wavelet techniques. In this paper the iris recognition approach is implemented via many steps, these steps are concentrated on image capturing, enhancement and identification. Different types of edge detection mechanisms; Canny scheme, Prewitt scheme, Roberts scheme and Sobel scheme are used to detect iris boundaries in the eyes digital image. The implemented system gives adequate results via different types of iris images.
\end{abstract}

KEYWORDS

Iris Recognition, Biometrics Recognition, Wavelet Technology, Hybrid Technique and Feature Extraction.

\section{INTRODUCTION}

Electronic arena is witnessing rapid sophisticated, a large and important. Recognition systems have become a role of the large and effective, especially after the progress that has occurred in the area of Information Technology. Iris is the main important part of the human eye; it consists of circular muscle and the other longitudinal control in the amount of light passing the retina through the human eye, with the increasing of requirements for higher security level, biometric systems have been widely used for many applications. Biometrics includes face, iris, fingerprints, voice, palms, hand geometry, retina, handwriting, gait etc. [3].

Recognition algorithms usually require a combination of various techniques, which span across all the three levels. At the lowest possible level are methods for localizing the region of concern in the image (background subtraction), detection and tracking of feature points, various morphological operations that may be needed in order to obtain a better quality of the input data, etc.

Biometric systems work by capturing of the feature taking a digital image for iris recognition. A biometric is characterized by use of a feature that is decidedly unique - so that the chance of any two human having the same features will be minimal. Will be used several techniques in our paper for high proportion of excellence by using the iris of the eye [1, 2]. However the importance of research in this area has increased the popularity of this system. Person identification based on iris recognition gives one of the most reliable results [11]. Iris texture features provides a unique high dimensional information that explains why iris recognition based 
verification has the lowest false acceptance rate among all types of biometric verification systems $[12,13]$.

\section{BIOMETRIC SYSTEM}

A biometric system is fundamentally a pattern-recognition system that recognizes a individual based on a attribute vector derived from a specific physiological or behavioral characteristic that the person possesses. That feature vector is frequently stored in a database (or recorded on a smart card given to the individual) after being extracted. A biometric system based on physiological characteristics is normally more reliable than one which adopts behavioral characteristics, even if the last may be easier to integrate within certain specific application. Biometric system can than run in two modes: verification or identification. While recognition involves comparing the acquired biometric information against templates corresponding to all users in the database, verification involves comparison with only those templates corresponding to the claimed identity. This implies that identification and verification are two problems that should be dealt with separately.

A simple biometric system consists of four basic components [14,15]:

- Sensor module witch acquires the biometric data.

- Feature extraction module where the acquire data is processed to extract feature vectors.

- Matching module where attribute vectors are compared against those in the template.

- Decision-making module in which the user's identity is established or a claimed identity is accepted or rejected.

Any human physiological or behavioral trait can serve as a biometric characteristic as long as it satisfies the following requirements [16]:

- Universality: Everyone should have it.

- Distinctiveness: No two should be the same.

- Permanence. It should be invariant over a given era of time.

- Collectability: In real life applications, three extra factors should also be considered: performance (accuracy, speed, resource requirements), acceptability (it must be harmless to users), and circumvention (it should be robust enough to various fraudulent methods).

\section{IRIS RECOGNITION}

The critical attributes for any biometrics are: the number of degree-of-freedom of variation in the chosen index across the human population, since this determines uniqueness; its immutability over time and its immunity to intervention; and the computational prospects for efficiently encoding and reliably recognizing the identifying pattern. In the whole human population, no two irises are alike in their mathematical detail, even among identical (monozygotic) twins. The probability that two irises could produce exactly the same Iris Code is approximately 1 in 1078 . (The population of the earth is around 1010.)

Iris recognition is a method of biometric authentication, based on extraction features of the iris of an individual's eyes. Each individual has a unique iris; the variation even exists between identical twins and between the left and right eye of the same person [7]. 


\section{RELATED WORKS}

Recent noticeable studies in personal identification based on the patterns and colors of the iris, below some at these researches:

Daugman implemented the system using 2-D Gabor wavelet filter for localization of iris, Gaussian transform for feature extraction, and 256-byte iris code for computation. The major contribution of Daugman is to provide statistical theories for degree of iris code agreement [4].

Boles et al. implemented the system that operated on the set of 1-D signals and obtaining the zero-crossing representations of these signals. The major idea of this system is to represent the features of the iris by fine-to-coarse approximations at different resolution levels based on the wavelet transform zero-crossing representation. The prototypes also have the advantage of processing 1-D iris signatures rather than 2-D images used in both [5].

Wildes et al. introduced recognition system that consists of an image acquisition rig (low light video camera, lens, frame grabber, diffuse polarized illuminator, and reticle for operator positioning) interface to a Sun SPARCstation20. This work is concentrated on the grabbing the images of iris and making routine procedures of iris recognition system efficient by applying Laplacian pyramid and hierarchical gradient-based image registration algorithm in pattern matching [6].

Jaemin Kim1 and et al. described a new iris recognition algorithm, which uses a low level of details. Combining statistical classification and elastic boundary fitting, the iris is first localized. Then, the localized iris image is down-sampled by a factor of $\mathrm{m}$, and filtered by a modified Laplacian kernel. Since the output of the Laplacian operator is sensitive to a small shift of the full-resolution iris image, the outputs of the Laplacian operator are computed for all space-shifts [8].

Xiaofu He and et al. proposed a novel fake iris detection method based on wavelet packet transform. First, wavelet packet decomposition is used to extract the attribute values which provide unique information for discriminating fake irises from real ones. Second, to enhance the detecting accuracy of fake iris, Support vector machine (SVM) is used to characterize the distribution boundary based on ex-tracted wavelet packet features, for it has good classification performance in high dimensional space and it is originally developed for two-class problems [9].

JialieShen and et al. developed an accurate gait recognition from video is a complex presses involving heterogeneous features. This article introduces a novel framework, called GC2F, for effective and efficient gait recognition and classification. Adopting a"refinement-andclassification" principle, the framework comprises two components: 1) a classier to generate advanced probabilistic features from low level gait parameters ; and 2) a hidden classier layer (based on multi layer perceptron neural network) to model the statistical properties of different subject classes [10].

Padma Polash Paul and et al. presented an iris recognition system in order to verify together the uniqueness of the person iris and also its performance as a biometric identification. A biometric system provides automatic identification of an individual based on a unique feature or characteristic possessed by the individual. Iris recognition is regarded as the most reliable and accurate biometric identification system available. The iris recognition system consists of an automatic segmentation system that is based on the Hough transform, and is able to focus the circular iris and pupil region, occluding eyelids and eyelashes, and reflections [17].

Bhawna chouhan and et al. provided a biometric system based on automatic identification of an person based on a unique attribute or characteristic possessed by the individual. Iris recognition is 
regarded as the most reliable and accurate biometric recognition system available. Especially it focuses on image segmentation and feature extraction for iris recognition process. The performance of iris recognition system highly depends on edge detection. The Canny Edge Detector is one of the most commonly used image processing tools, detecting edges in a very robust manner. For instance, even an effective feature extraction method would not be able to obtain useful information from an iris image that is not segmented properly [18].

Jing Dong and et al. studied two application scenarios in the context of iris recognition, namely protection of iris templates by hiding the min cover images as watermarks (iris water marks), and protection of iris images by watermarking them. Experimental results suggest that water mark embedding in iris images does not introduce detect able decreases on iris recognition performance where as recognition performance drops significantly if iris water marks suffer from severe attacks [19].

\section{IMPLEMENTED IRIS RECOGNITION SYSTEM}

Biometric recognition refers to an automatic recognition of individuals based on a attribute vector(s) derived from their physiological and/or behavioral feature. Biometric iris recognition systems should provide a reliable personal recognition schemes to either confirm or determine the identity of an person. Different algorithms are implemented to perform iris recognition system. In this work, iris recognition system is implemented via hybrid technique. This system consists of the following components as illustrated in figure (1):

Step 1: Preprocessing \& Iris capturing: this process includes revises processing in which the image must be adequate to be adaptive to the next step. Then the iris it truncated and resizing from the original image.

Step 2: Converting image into gray scale: this process deals with the converting of color image into gray scale image.

Step 3: Histogram equalization: this process includes redistributing of pixels in order to enhance the overall image.

Step 4: 2D DWT: this process in applied to generate the most significant features of the image in order to minimize the processing time as well as to get minimum reduction size.

Step 5: Edge detection: this process in useful to generate the minimum feature required to identify the specific iris.

Step 6: Storage process: this process tries to arrange the generated features into vector to be ready in testing processing. 


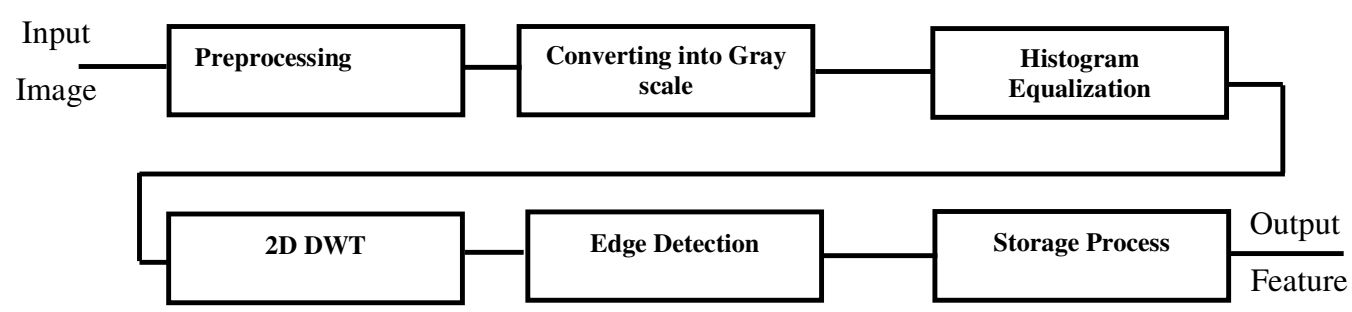

Figure 1 Iris recognition systems

\section{RESUltS AND ANALYSIS}

The implemented iris recognition system is tested via different types of images. This section tries to visualize the implemented results to ensure the effect of each step.

Step 1: Preprocessing \& Iris capturing

This step is applied to the original image in which the image is filtering and capturing to specify the boundaries of iris image. Preprocessing step is performed to minimize noise as possible as well as to resizing the original image as shown in figure (2).

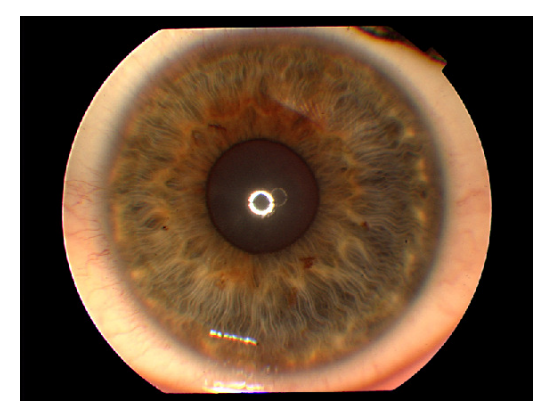

Figure 2 Capturing the original color image

Step 2: Converting Image into Gray Scale

This step is used to convert the original image into gray scale image as illustrated in figure (3)

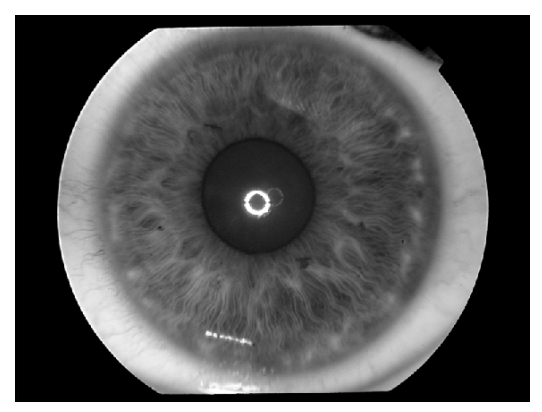

(a) Gray image and contains the noise

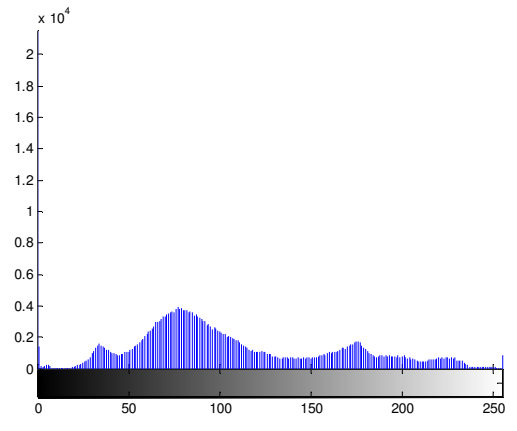

(b) Histogram of the gray image

Figure 3 converting the original color image into gray scale image 
Step 3: Enhance Iris Image

Two types of enhancement are used to improve image quality:

Histogram equalization is used to enhance the iris image and to improve its equalization as shown in figure (4).
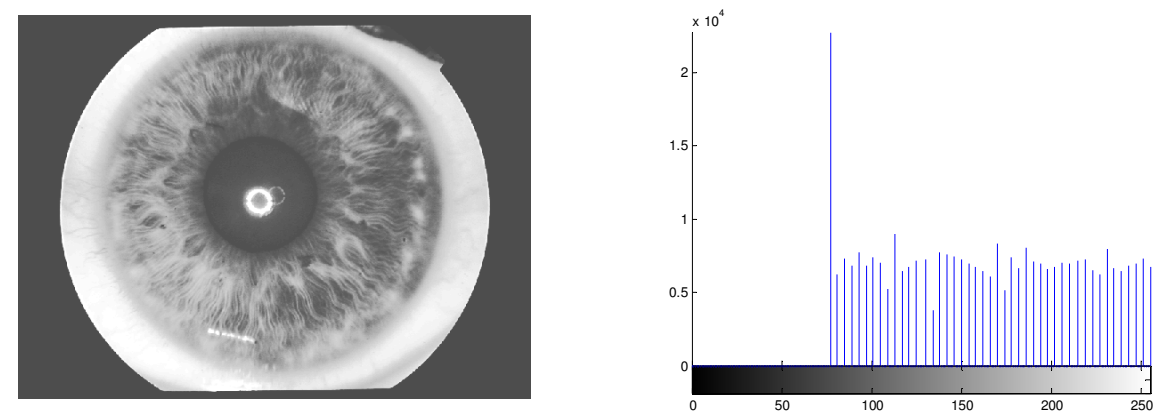

(a) Enhanced image

(b) Histogram of the enhanced image

Figure 4 Image enhancement using histogram equalizing

Then adaptive histogram equalization is used to enhance the iris image and to improve its equalization as shown in figure 5 .

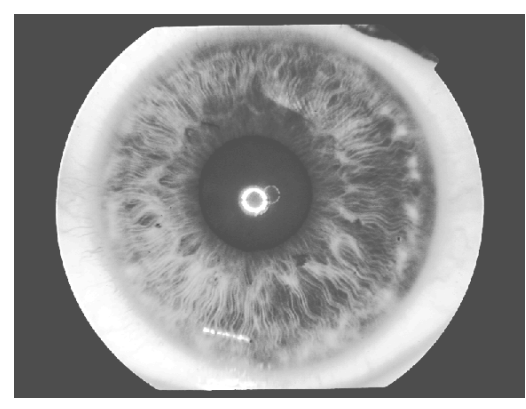

(a) Enhanced image

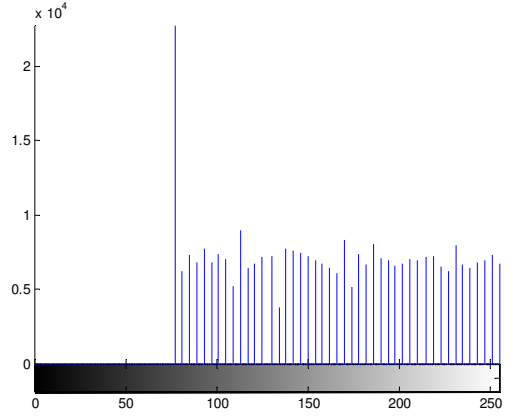

(b) Histogram of the enhanced image Figure 5 Image enhancement using histogram equalizing

Step 4: Two Dimensional Discrete Wavelet Transform To generate the specific features we applied 2D DWT of the enhanced image in which the output image is compressed to generate minimum number of suitable pixels as shown in the figure (6) and figure (7). In this step there are many wavelet masks, in addition we applied only two types which is Harr and Db2 wavelet transform masks which give an adequate results of feature extraction. 
International Journal on Soft Computing ( IJSC ) Vol.2, No.4, November 2011

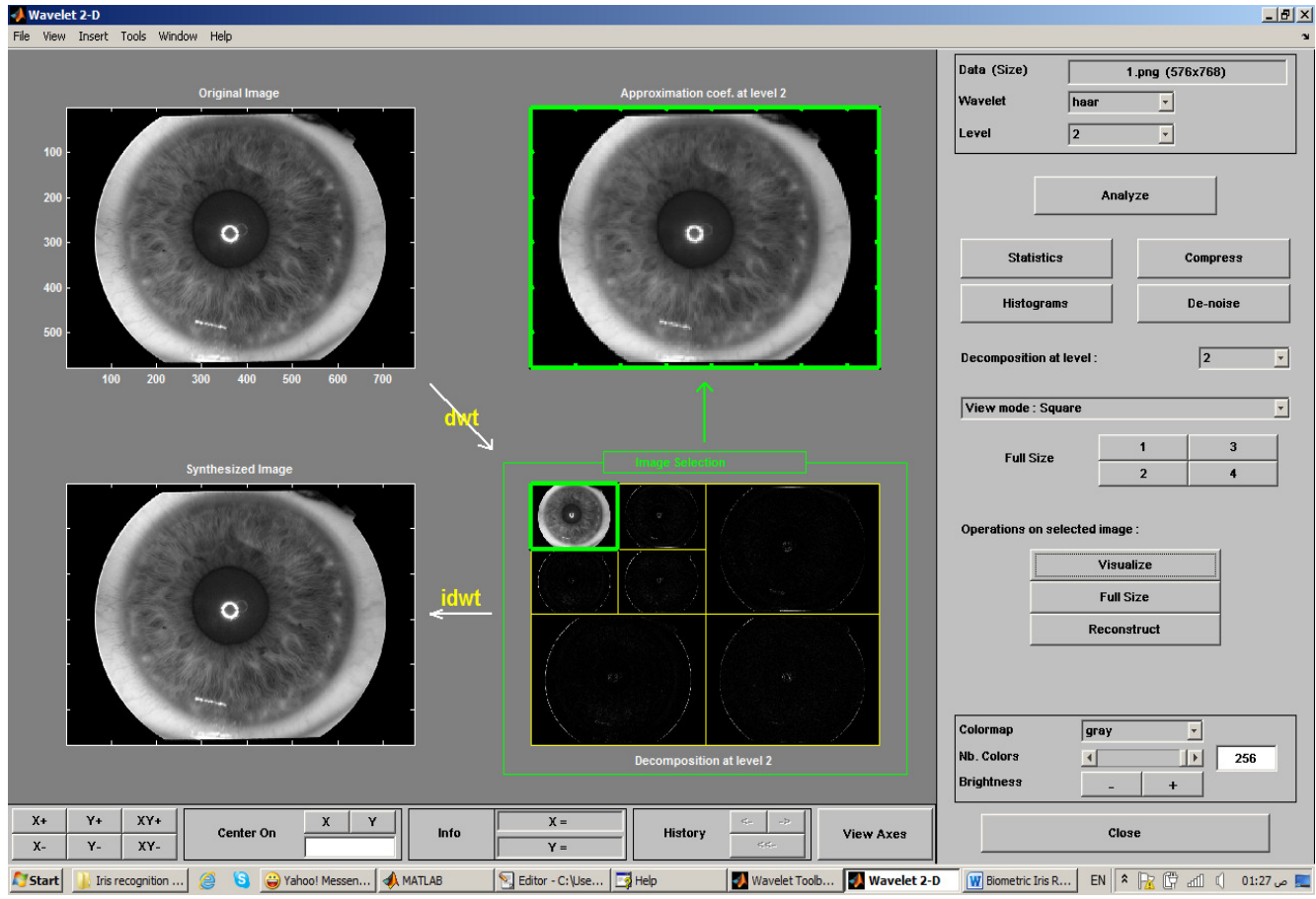

Figure 6 Two levels of two dimensional Harr discrete wavelet transform

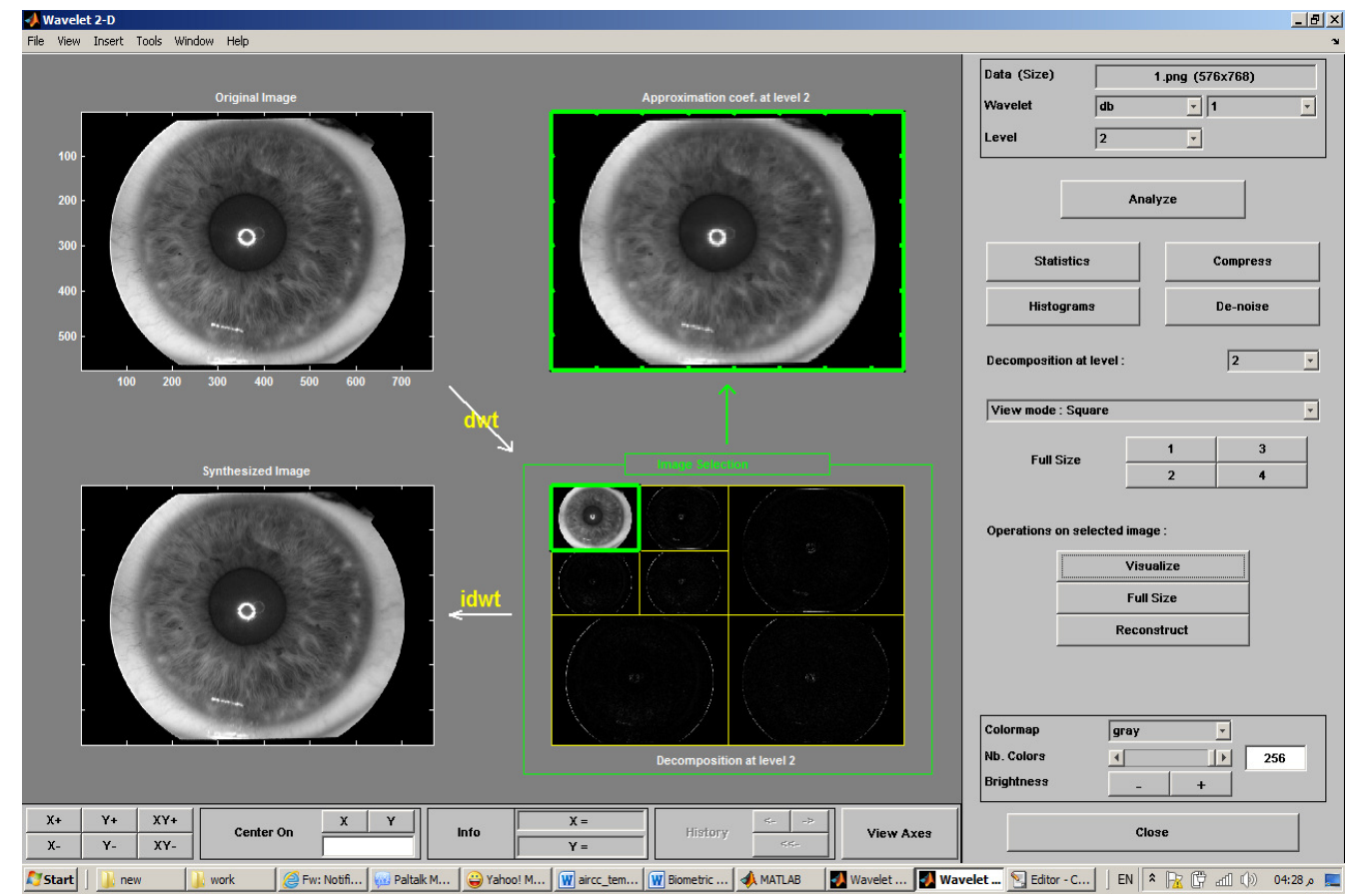

Figure 7 Two levels of two dimensional Db2 discrete wavelet transform

Step 5: Edge detection

Different type of edge detection makes are applied to recognize the iris features; Canny, Prewitt, Roberts and Sobel as shown in figure (7) (a), (b), (c) \& (d). It is clear that the obtained results of b, c \& d are approximately similar, but the result of a leads to different details, this is due to high power of Canny mask. 


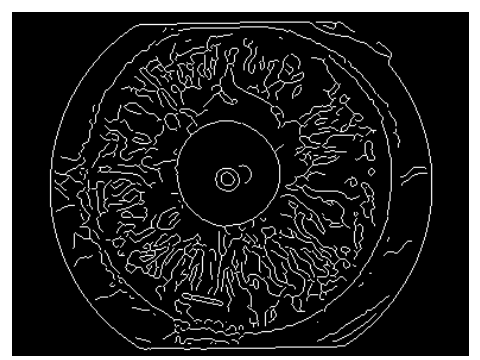

(a) Canny method

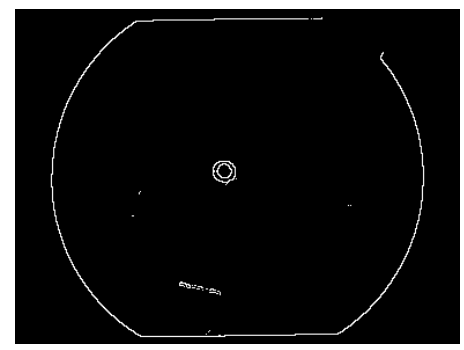

(c) Roberts method

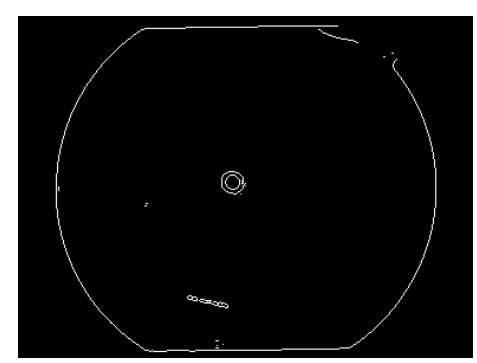

(b) Prewitt method

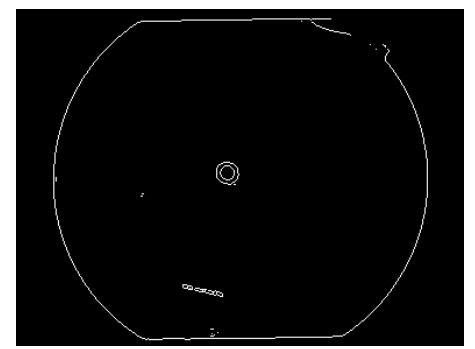

(d) Sobel method

Figure 7 Edge detection applications

\section{Conclusion}

Biometrics refers to an automatic recognition of a individual based on her behavioral and/or physiological features. Many business applications will in future rely on biometrics since using biometrics is the only way to guarantee the presence of the owner when a transaction is made. Iris recognition systems have been proven to be very effective in protecting information and resources in a large area of applications. Biometric iris recognition system is implemented via wavelet transform and edge recognition techniques. This approach leads to high performance compared with many separated systems.

\section{Acknowledgements}

I would like to express my gratitude to Dr. Muzhir Shaban Al-Ani for his help and assistance throughout the development of this research.

\section{REFERENCES}

[1] Jain, A.K., Bolle, R.M., Pankanti, S. (eds.): Biometrics: Personal Identification in Net-worked Society. Kluwer, Norwell (1999).

[2] Prabhakar, S., Kittler, J., Maltoni, D., O'Gorman, L., Tan, T.: Introduction to the SpecialIssue on Biometrics: Progress and Directions. IEEE Trans. Pattern Anal. Mach. Intell. 29(4), 513-516 (2007).

[3] Xiaofu He and et al," A New Fake Iris Detection Method", M. Tistarelli and M.S. Nixon (Eds.): ICB 2009, LNCS 5558, pp. 1132-1139, 2009. Springer-Verlag Berlin Heidelberg 2009.

[4] John G. Daugman, "High Confidence Visual Recognition of Persons by a Test of Statistical Independence", IEEE Trans. on Pattern Analysis and Machine Intelligence, 15(11), pp. 1148-1161, 1993. 
[5] Boles, W.W.; Boashash, B., "A Human Identification Technique Using Images of the Iris and Wavelet Transform", IEEE Trans. on Signal Processing, 46(4), pp.1185-1188, 1998.

[6] Wildes, R.P., Asmuth, J.C., et.al, "A System for Automated Iris Recognition", Proc. of the Second IEEE Workshop on Applications of Computer Vision, pp.121- 128, 1994.

[7] Wildes, R.P, "Iris Recognition: An Emerging Biometric Technology", Proceedings of the IEEE, VOL. 85, NO. 9, September 1997, pp. 1348-1363.

[8] Jaemin Kim and et al," Iris Recognition Using a Low Level of Details", School of Electronics and Electrical Engineering, Hongik University,72-1 Sangsu-dong, Mapo-gu, Seoul 121-791, Korea, 2006.

[9] Xiaofu He and et al," A New Fake Iris Detection Method", Department of Computer Science and Technology, East China Normal University, Shanghai 200241, China, 2009.

[10] JialieShen and et al," Dual Phase Learning for Large Scale Video Gait Recognition", School of Information Systems, Singapore Management University, (C) Springer-Verlag Berlin Heidelberg 2010.

[11] Algirdas Bastys," Iris Matching by Local Extremum Points of Multi scale Tayl or Expansion", ICB2009, LNCS5558, pp.1070-1079, Springer-Verlag Berlin Heidelberg, 2009.

[12] Daugman, J., Dowing, C. "Epigenetic randomness, complexity, and singularity of human iris patterns. In: Preceding soft the Royal Society, B, 268, Biological Sciences, pp.1737-1740(2001).

[13] Daugman, J." Statistical richness of visual phase information: update on recognizing Persons by iris patterns. Int. J. Comput.Vis.45 (1), 25-38(2001).

[14] 6th International Symposium Electronics in Marine, ELMAR-2004, 16-18 June 2004, Zadar, Croatia 185.

[15] S. Prabhakar, S. Pankanti, A. K. Jain, "Biometric Recognition: Security and Privacy Concerns", IEEE Security \& Privacy, March/April 2003, pp. 33-42.

[16] A. K. Jain, A. Ross, S. Prabhakar, "An Introduction to Biometric Recognition", IEEE Trans. on Circuits and Systems for Video Technology, Vol. 14, No. 1, pp 4-19, January 2004.

[17] Padma Polash Paul*, Md. Maruf Monwar," Human Iris Recognition for Biometric Identification",Ahsanullah University of Science and Technology, Dhaka, Bangladesh, 2007.

[18] Bhawna chouhan, shailja shukla." Iris Recognition System using canny edge detection for Biometric Identification", Interrnational Journal of engineering Sciences and Technology (IJEST), ISSN: 0975 5462 Vol. 3 No. I Jan 2011.

[19] JingDong, TieniuTan," Effects of Watermarking on Iris Recognition Performance", 10th Intl Conf. on Control, Automation, Robotics and Vision, Hanoi, Vietnam, 17-20 December 2008.

\section{Author}

Khattab M. Ali was born in Anbar-Iraq at 1978. He received his B.Sc. from Al_M'ammon college university at 2000, Baghdad, Iraq. He received MSc. degree from CS Department in Al-Bayt University, Jordan 2008. He joined in 5 January 2005 Computer Sciences Department, Almaref College University, AlAnbar, Iraq. Since 2009 till now he joined as Assistant Instructor at the college of computer, Information System Department, Al-Anbar University, Iraq.

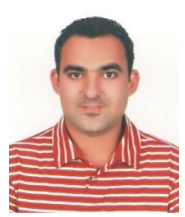

